\title{
Spatial-multiplexed Dual-view Three-dimensional Display Based on One-dimensional Integral Imaging
}

\author{
Zhaoxu Ren ${ }^{\mathrm{a}}$, Rui Wu ${ }^{\mathrm{b}}$ \\ Chengdu Technological University, \\ Chengdu 611730, China \\ awiston_ren@sina.cn, b15982227662@163.com
}

\begin{abstract}
We propose a spatial-multiplexed dual-view one-dimensional integral imaging (DV1DII) three-dimensional (3D) display which presents different 3D images in the left and right viewing directions simultaneously. The DV1DII 3D display consists of a display panel, a parallax barrier and two polarizer parallax barriers. The elemental images displayed on the left and right half of the display panel are captured from two different 3D scenes, respectively. The lights emitted from two kinds of elemental images are modulated by the left and right half of the parallax barrier to present two different 3D images, respectively. A prototype of the DV1DII 3D display is developed, and good results are obtained.
\end{abstract}

Keywords-spatial-multiplexed; dual-view; integral imaging; three-dimensional display

\section{INTRODUCTION}

Multi-view display which presents multiple images to more than one user on one screen is regarded as a promising display. Users see different information from different viewing directions on the multi-view display which saves considerable space and cost. For example, in a car, the multi-view display presents live traffic information to the driver and meanwhile presents NBA games to the passenger sitting in the co-pilot position. The multi-view display has been implemented by using a display panel and additional parallax barrier or lenticular lens array. Moreover, a few methods using single display panel are proposed to realize the multi-view display [1-3]. A dual-view liquid crystal display fabricated by patterned electrodes is proposed by adopting spatial-multiplexed. The main pixel in the dual-view liquid crystal display is composed of a left sub-pixel and a right sub-pixel which have the opposite rotation directions. Two different images are presented to different viewers who are viewing at different directions. Blue phase liquid crystal combined with a directional backlight module realizes dual-view display by adopting time-multiplexed. By controlling the light sources and image data, the time-multiplexed dual-view display displays two different images to dif-ferent viewers sequentially. However, those methods present only two-dimensional (2D) images to the observers.
Integral imaging (II), which was first proposed by Lippmann in 1908, is one of the most promising and feasible 3D display methods [4-7]. A one-dimensional in-tegral imaging (1DII) reduces ray information by removing vertical parallax and provides three-dimensional (3D) images with high resolution in the vertical direc-tion. Although 1DII resembles multiview, an important difference between the 1DII display and the multiview autostereoscopic display is defined in terms of the position and the interval of beam condensing points. There is no need to predeter-mine the observer's viewpoints in the 1DII display. However, the observer's viewpoints are light ray converging points and an important design parameter in the multiview autostereoscopic display. The 1DII displays have been implemented by use of a parallax barrier or a lenticular lens array. The 1DII display based on a parallax barrier has less space and lower cost than that based on a lenticular lens array. So, we propose a spatial-multiplexed dual-view one-dimensional integral imaging (DV1DII) display which presents two different $3 \mathrm{D}$ images in the left and right viewing directions.

\section{PRINCIPLE AND STRUCTURE}

The principle and structure of the DV1DII 3D display are shown in Figure 1. It con-sists of a display panel, a parallax barrier and two polarizer parallax barriers (PPBs). PPB 1 is in front of the display panel, and PPB 2 is in front of the parallax barrier. The centers of the PPB1 and PPB2 are located at the centers of the display panel and the parallax barrier, respectively. As shown in Fig. 1, two kinds of diagonals represent horizontal and vertical polarization directions of the image light, respectively. The central unit of PPB 2 consists of two parts with perpendicular polarization directions, whereas others units of PPB 1 and PPB 2 have only horizontal or vertical polarization directions. To eliminate the flipping images in the left-view and right-view 1DII 3D displays, the polarization directions of adjacent elements in PPB1 and PPB2 are orthorhombic. The EIs displayed on the left and right half of the display panel are denoted as left elemental images (LEIs) and right elemental images (REIs), respectively. The LEIs and REIs are captured from two different 3D scenes, respectively. The lights emitted from the LEIs are modulated by the left half of the parallax barrier and propagated to reconstruct a $3 \mathrm{D}$ image in 
right-view area, whereas the lights emitted from the REIs are modulated by the right half of the parallax barrier and propagate to the reconstruct another 3D image in left-view area. Thus, two different 3D images are simultaneously obtained in the left-view and right-view 1DII 3D displays.

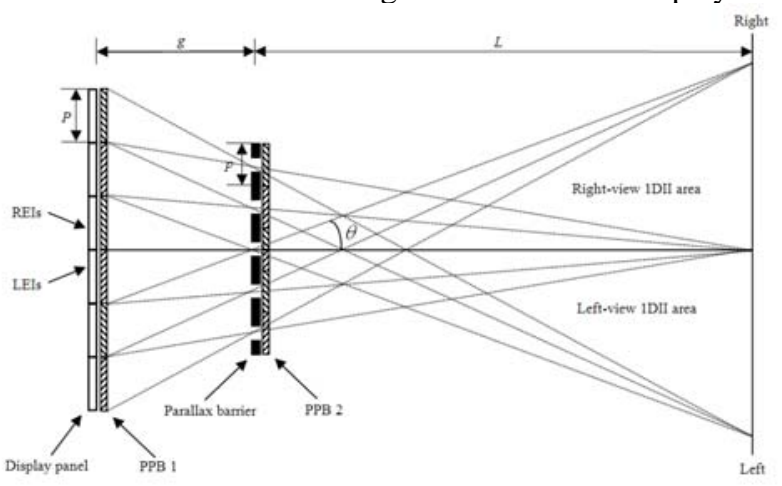

Figure 1. Principle and structure of the DV1DII 3D display.

Suppose that $g$ is the gap between the display panel and the parallax barrier, $P$ is the pitch of the EI, $p$ is the pitch of the parallax barrier, and $L$ is the optimal viewing distance between the parallax barrier and the observer. At the optimal viewing distance, the viewing angle of each II 3D display $\theta$ is shown as [8]

$$
\theta=\arctan \left(\frac{P}{g}\right) \text {. }
$$

As shown in Fig. 1, when the viewing distance between the parallax barrier and the observer is smaller or larger than $L$, a flipping zone appears between the left-view zone and right-view zone, and the viewing angle of each II display is decreased. Therefore, $L$ is an important parameter in the DV1DII 3D display. Based on the geometric relationships in Fig. 1, the optimal viewing distance $L$ is obtained as [9]

$$
L=\frac{P g}{P-p} \text {. }
$$

Since the DV1DII 3D display is usually used in a car, the positions of the DV1DII 3D display and the front seats are fixed. Therefore, the distance between the DV1DII 3D display and the front seats in the car is fixed as $L$. From Eq. (2), we can see that the DV1DII 3D display can satisfy different needs by adjusting $P, p$ and $g$.

\section{EXPERIMENTAL RESULTS}

A prototype of the DV1DII 3D display is developed. In our experiment, a back light unit and a film were combined as a display panel with high resolution. The display panel was used to display the EIA for the DV1DII 3D display. Another film was used as the parallax barrier of the DV1DII 3D display. Each PPB is composed of a striped half wave plate, a diffusion screen, and a striped polarizer [10]. The gap between the display panel and the parallax barrier is $3 \mathrm{~mm}$. The pitched of the EI and the parallax barrier are $2.5 \mathrm{~mm}$, and $2.4 \mathrm{~mm}$, respectively. The optimal viewing distance between the parallax barrier and the observer is $75 \mathrm{~mm}$.

The LEIs and REIs of the DV1DII 3D display were captured from two view zones for two different scenes by using a computer. The 3D scene of the left-view 1DII 3D display includes two letters SC, and the 3D scene of the right-view 1DII 3D display includes two letters CS. The EIA of the DV1DII 3D display was generated by using Matlab software, as shown in Figure 2.

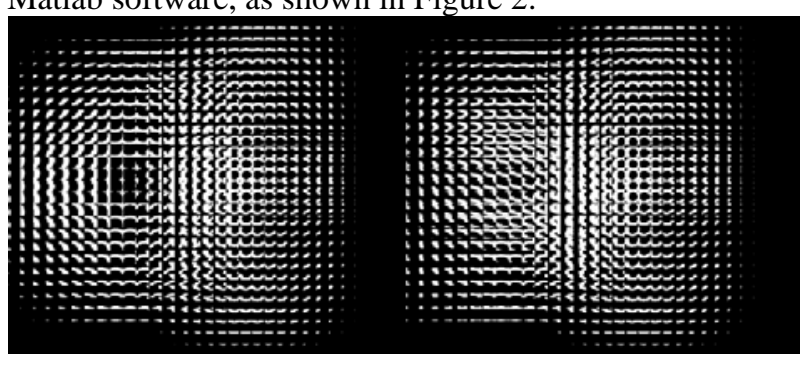

Figure 2. EIA of the DV1DII 3D display.

Corresponding to the parameter of the DV1DII 3D display, the theoretical viewing angle of each 1DII 3D display is $39^{\circ}$ at the optimal viewing distance. However, in practice, the slit isn't an ideal point and the aperture is 0.1 $\mathrm{mm}$. Therefore, the left-view and right-view 1DII displays are slightly decreased and the practical viewing angle of each II 3D display is $36^{\circ}$ [11]. 3D images viewed from different angles in the DV1DII 3D display are shown in Figure 3 . When the viewing angle is $36^{\circ}$ to the left, the two letters SC are integrated in the left-view 1DII display, as shown in Figure 3 (a). Decreasing the viewing angle to $3^{\circ}$ to the left, the relative positions of the two letters SC are changed, as shown in Figure 3 (b). When the viewing angle is $3^{\circ}$ to the right, two letters CS are integrated in the right-view 1DII display, as shown in Figure 3 (c). Increasing the viewing angle to $36^{\circ}$ to the right, the relative positions of the two letters CS are also changed, as shown in Figure 3 (d). Therefore, two different 3D images are obtained in the left-view and right-view 1DII displays, simultaneously.

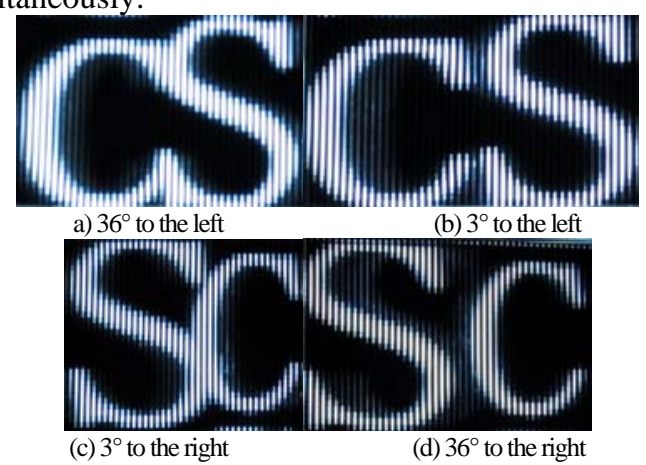

Figure 3. 3D images captured from different angles in the DV1DII 3D display. 


\section{CONCLUSIONS}

A spatial-multiplexed DV1DII 3D display is proposed. The DV1DII 3D display consists of a display panel, a parallax barrier and two polarizer parallax barriers. The LEIs and REIs are captured from two view zones for two different 3D scenes, respectively. By using two polarizer parallax barriers, the lights emitted from left and right element images are modulated by the left and right half of the parallax barrier to present the right-view and left-view 1DII 3D displays, respectively. A prototype of the DV1DII 3D display using parallax barrier is developed, and two different 3D images are obtained in the left and right viewing directions, simulta-neously. It has great potential application in 3D displays.

\section{ACKNOWLEDGEMENTS}

The work was financially supported by the Scientific Research Fund of Sichuan Provincial Education Department under Grant (14ZB0292).

\section{REFERENCES}

[1] G. Lippmann: C. R. Acad. Sci. Vol.146 (1908) p.446-451.

[2] J. S. Jang, and B. Javidi: Opt. Lett. Vol. 27 (2002) p.324-326.

[3] F. Wu, H. Deng, C. G. Luo, D. H. Li, and Q. H. Wang: Appl. Opt. Vol. 52 (2013) p.4911-4914.

[4] B. Lee, S. Jung, and J. H. Park: Opt. Lett. Vol. 27 (2002) p.818-820.

[5] Y. Kim, J. H. Park, H. Choi, J. Kim, S. W. Cho, and B. Lee: Appl. Opt. Vol. 45 (2006) p.4334-4343.

[6] Y. Kim, H. Choi, J. Kim, S. W. Cho, Y. Kim, G. Park, and B. Lee: Appl. Opt. Vol. 46 (2007) p.3766-3773.

[7] H. Kim, J. Hahn, and B. Lee: Opt. Express Vol. 16 (2008) p.21865-21880.

[8] C. G. Luo, C. C. Ji, F. N. Wang, Y. Z. Wang, and Q. H. Wang: J. Display Technol. Vol. 8 (2012) p.634-638.

[9] F. Wu, H. Deng, C. G. Luo, D. H. Li, and Q. H. Wang: Chin. Opt. Lett. Vol. 11 (2013) p.071101.

[10] F. Wu, H. Deng, D. H. Li, and Q. H. Wang: Opt. Eng. Vol. 52 (2013) p.093102. 